\title{
A Quadratic Constraint Total Least-squares Algorithm for Hyperbolic Location
}

\author{
Kai YANG', Jianping AN, Zhan XU \\ Department of Electronic Engineering, School of Information Science and Technology, \\ Beijing Institute of Technology, Beijing 100081, P.R. China \\ E-mail: 1yangkbit@gmail.com
}

\begin{abstract}
A novel algorithm for source location by utilizing the time difference of arrival (TDOA) measurements of a signal received at spatially separated sensors is proposed. The algorithm is based on quadratic constraint total least-squares (QC-TLS) method and gives an explicit solution. The total least-squares method is a generalized data fitting method that is appropriate for cases when the system model contains error or is not known exactly, and quadratic constraint, which could be realized via Lagrange multipliers technique, could constrain the solution to the location equations to improve location accuracy. Comparisons of performance with ordinary least-squares are made, and Monte Carlo simulations are performed. Simulation results indicate that the proposed algorithm has high location accuracy and achieves accuracy close to the CramerRao lower bound (CRLB) near the small TDOA measurement error region.
\end{abstract}

Keywords: Location, Time Difference of Arrival, Total Least-squares

\section{Introduction}

Determining the location of a source from its emissions is a critical requirement for the deployment of wireless sensor networks in a wide variety of applications [1]. Location finding based on time difference of arrival (TDOA), which does not require knowledge of the absolute transmission time, is the most popular method for accurate positioning systems [2]. The idea of TDOA is to determine the source location relative to the sensors by examining the difference in time at which the signal arrives at multiple measuring sensor units, rather than the absolute arrival time. Sensors at separate locations measuring the TDOA of the signal from a source can determine the location of the source as the intersection of hyperbolae for TDOA. For each TDOA measurement, the source lies on a hyperbola with a constant range difference between the two measuring sensors. However, finding the solution to the hyperbolic location equations is not easy as the equations are nonlinear. Furthermore, the nonlinear hyperbolic equations become inconsistent as errors occur in TDOA measurements and the hyperbolae no longer intersect at a single point.

In the past, the source location determination problem has been mathematically formulated as a set of linear equations $\mathbf{A x}=\mathbf{b}$ which is in matrix form and ordinary least-squares (OLS) technique is utilized to find the maximum-likelihood solution by assuming the system matrix $\mathbf{A}$ is error-free and all errors are confined to the data vector b [3-6]. However, in the TDOA based location problem there are errors in both system matrix $\mathbf{A}$ and data vector $\mathbf{b}$. Using OLS technique for this problem will result in biased solution and location accuracy will decrease due to the accumulation of the system matrix errors. To alleviate this problem, a generalization of the OLS solution, called total leastsquares (TLS) [7-9], is utilized to remove the noise in $\mathbf{A}$ and $\mathbf{b}$ using a perturbation on $\mathbf{A}$ and $\mathbf{b}$ of the smallest Frobenius norm which makes the system of equations consistent. It is shown that in the TDOA based location problem the unknown parameters in vector $\mathbf{x}$ are quadratic constraint related, which could be realized via Lagrange multipliers technique to constrain the TLS solution.

For practical applications, the location estimation algorithm should be robust and easy to implement, and the source location estimation should minimize its deviation from the true location. In this paper, a novel TDOA based QC-TLS algorithm for source location 
problem is presented. The rest of the paper is organized as follows. The formulation of TDOA based location estimation problem is described in Section 2. In Section 3 , this problem is shown to be in the form of an overdetermined set of linear equations with errors in both $\mathbf{A}$ and $\mathbf{b}$ and the QC-TLS algorithm is applied to the TDOA measurement data for source location estimation. Computer simulations are used to verify the validity of the algorithm and simulation results are presented in Section 4, which is followed by conclusions.

\section{Problem Formulation}

There are two basic ways to measure the TDOA in wireless sensor networks: the direct way and the indirect way. In the direct way, we could obtain the TDOA through the use of cross-correlation techniques, in which the received signal at one sensor is correlated with the received signal at another sensor. The timing requirement for this method is the synchronization of all the receivers participating in the TDOA measurements, which is more available in location applications. However, in multipath channels there is ambiguity in detecting the TDOA using cross-correlation techniques since the correlation peak to be detected may not be caused by the two direct line of sight (LOS) paths [10]. In the indirect way, we first obtain the arrival time of received signal transmitted from the source at spatially separated sensors and then subtract the measurements of arrival time at two sensors to produce a relative TDOA. TDOA also could be obtained by subtracting absolute TOA measurements, but this requires the synchronization of source and sensors. In this paper, because of these factors above we obtain the TDOA by subtracting the measurements of arrival time at two sensors.

When TDOA based location method is adopted to give source location estimation in wireless networks, according to TDOA measurements a set of hyperbolic equations is given by

$$
\begin{aligned}
& r_{i 1}=c \tau_{i 1}=r_{i}-r_{1}, i=2,3, \cdots, M \\
& r_{i}=\sqrt{\left(x_{i}-x_{\mathrm{s}}\right)^{2}+\left(y_{i}-y_{\mathrm{s}}\right)^{2}}
\end{aligned}
$$

where $c$ is the speed of signal propagation, $\tau_{i 1}$ is the true value of TDOA measurement between sensor $i$ and $1, r_{i}$ is the distance between the source and sensor $i, M$ is the number of sensors, and $\left(x_{\mathrm{s}}, y_{\mathrm{s}}\right)$ and $\left(x_{i}, y_{i}\right)$ are the coordinates of the source and sensor $i$ respectively.

Solving those nonlinear equations is difficult. Linearizing them and then solving is one possible way. From (1), we have

$$
r_{i 1}+r_{1}=r_{i}
$$

Substituting the expressions of $r_{i}$ and $r_{1}$ into (2) and then squaring both sides of (2) produces

$$
\begin{aligned}
& \left(x_{i}-x_{1}\right)\left(x_{\mathrm{s}}-x_{1}\right)+\left(y_{i}-y_{1}\right)\left(y_{\mathrm{s}}-y_{1}\right)+r_{i 1} \cdot r_{1} \\
= & \frac{1}{2}\left(\left(x_{i}-x_{1}\right)^{2}+\left(y_{i}-y_{1}\right)^{2}-r_{i 1}^{2}\right)
\end{aligned}
$$

Formulation it in matrix form we have

$$
\mathbf{A x}=\mathbf{b}
$$

where

$$
\begin{aligned}
& \mathbf{A}=\left[\begin{array}{ccc}
x_{2}-x_{1} & y_{2}-y_{1} & r_{21} \\
x_{3}-x_{1} & y_{3}-y_{1} & r_{31} \\
\vdots & \\
x_{M}-x_{1} & y_{M}-y_{1} & r_{M 1}
\end{array}\right] \\
& \mathbf{x}=\left[\begin{array}{lll}
x_{s}-x_{1} & y_{s}-y_{1} & r_{1}
\end{array}\right]^{T} \\
& \mathbf{b}=\frac{1}{2}\left[\begin{array}{c}
\left(x_{2}-x_{1}\right)^{2}+\left(y_{2}-y_{1}\right)^{2}-r_{21}^{2} \\
\left(x_{3}-x_{1}\right)^{2}+\left(y_{3}-y_{1}\right)^{2}-r_{31}^{2} \\
\vdots \\
\left(x_{M}-x_{1}\right)^{2}+\left(y_{M}-y_{1}\right)^{2}-r_{M 1}^{2}
\end{array}\right]
\end{aligned}
$$

and the superscript $\mathrm{T}$ denotes the matrix transpose operation.

In the absence of noise and interference, hyperbolae from two or more sensors will intersect to determine a unique location which means that the overdetermined set of linear equations (4) is consistent. In the presence of noise, more than two hyperbolae will not intersect at a single point which means that (4) is inconsistent. In the following section, we will develop the solution to the overdetermined equations.

\section{Hyperbolic Location Solution}

In this section we first analyze the OLS solution to (4) and then give the QC-TLS solution.

\subsection{OLS Approach}

In our applications, the usual assumption for OLS approach is that the matrix A consists of $M^{-1}$ observations on each of the 3 independent variables. The dependent variable is represented by vector $\mathbf{b}$, in which we try keeping the correction term $\Delta \mathbf{b}$ as small as possible while simultaneously compensating for the noise present in $\mathbf{b}$ by forcing $\mathbf{A x}=\mathbf{b}+\Delta \mathbf{b}$ [7].

Under these assumptions the least-squares estimator

$$
\mathbf{x}=\left(\mathbf{A}^{T} \mathbf{A}\right)^{-1} \mathbf{A}^{T} \mathbf{b}
$$


in which it is implicit that $\mathbf{A}$ is known exactly. Suppose now that the elements of the A matrix contain errors. The system matrix A resulting from the calculation of (4) by using the measured values of TDOA can be given by $\mathbf{A}=\mathbf{A}_{0}+\mathbf{E}$, where $\mathbf{A}_{0}$ and $\mathbf{E}$ represent the true value and error respectively. Under the assumption that the error $\mathbf{E}$ is reasonably small, retaining only the linear error terms, the OLS estimator is given by [11]

$$
\mathbf{x}(\mathbf{E})=\mathbf{x}+\left(\mathbf{A}_{0}^{T} \mathbf{A}_{0}\right)^{-1} \mathbf{E}^{T} \boldsymbol{\eta}-\left(\mathbf{A}_{0}^{T} \mathbf{A}_{0}\right)^{-1} \mathbf{A}_{0}^{T} \mathbf{E} \mathbf{x}
$$

where $\boldsymbol{\eta}$ is the vector of residuals $\mathbf{b}-\mathbf{A x}$ which would be obtained if A were known accurately.

The sensitivity of each estimated parameter $x_{k}$ to each element $A_{j i}$ of system matrix A comes immediately from (6) by taking the derivative of $x_{k}$ with respect to $A_{j i}$ and this yields

$$
\frac{\partial x_{k}}{\partial A_{j i}}=\left(\mathbf{A}_{0}^{T} \mathbf{A}_{0}\right)_{k i}^{-1} \eta_{j}-\left(\sum_{l=1}^{3}\left(\mathbf{A}_{0}^{T} \mathbf{A}_{0}\right)_{k l}^{-1} A_{j l}\right) x_{i}
$$

The sensitivities are different, both in value and ranking, from the sensitivities to change in the dependent variable. It would in particular give details of those observations most liable to cause estimation error.

\subsection{QC-TLS Approach}

In hyperbolic location problem, it can be argued that both the system matrix $\mathbf{A}$ and vector $\mathbf{b}$ are subject to error, which is out of accord with the assumption of OLS. To

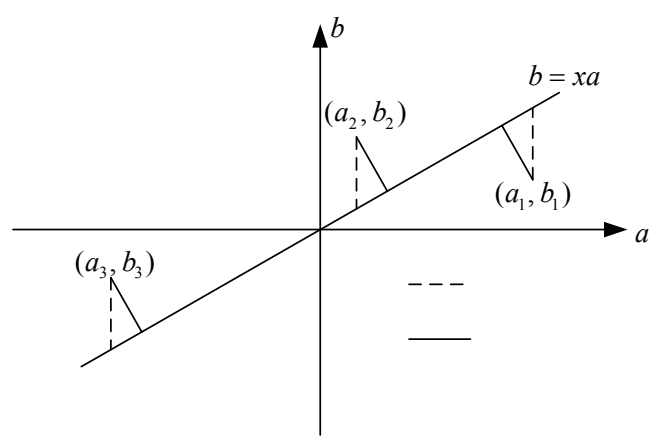

Figure 1. Least-squares versus total least-squares

alleviate the effects of these errors, we propose to use the TLS solution for the source location problem. The total least-squares method [8,9], which is a natural extension of LS when errors occur in all data, is devised as a more global fitting technique than the ordinary least-squares technique for solving overdetermined sets of linear equations by trying to remove the data errors. The LS and TLS measures of goodness are shown in Figure 1. In the LS approach, it is the vertical distances that are important; whereas in the TLS problem, it is the perpendicular distances that are critical. So, from this geometric interpretation, it's shown that the TLS method is better than the LS method with respect to the residual error in the curve fitting.

When errors exist in TDOA measurements, (4) can be represented by

$$
\left(\mathbf{A}_{0}+\mathbf{E}\right) \mathbf{x}=\mathbf{b}_{0}+\mathbf{w}
$$

where $\mathbf{E}$ and $\mathbf{w}$ are the perturbations of $\mathbf{A}$ and $\mathbf{b}$, respectively. And (8) also can be put into the following form

$$
\left(\left[-\mathbf{b}_{0} \vdots \mathbf{A}_{0}\right]+[-\mathbf{w} \vdots \mathbf{E}]\right)\left[\begin{array}{l}
1 \\
\mathbf{x}
\end{array}\right]=\mathbf{0}
$$

or

Where

$$
\left(\mathbf{B}_{0}+\mathbf{D}\right) \boldsymbol{\alpha}=\mathbf{0}
$$

$$
\mathbf{B}_{0}=\left[-\mathbf{b}_{0} \vdots \mathbf{A}_{0}\right], \mathbf{D}=[-\mathbf{w} \vdots \mathbf{E}], \boldsymbol{\alpha}=\left[\begin{array}{ll}
1 & \mathbf{x}^{T}
\end{array}\right]^{T}
$$

The rank of matrix $\mathbf{B}_{0}$ is three, because it is equal to the rank of matrix $\mathbf{A}_{0}$.

The TLS solution to this problem is to looks for the minimal (in the Frobenius norm sense) perturbation matrix $\mathbf{E}$ and perturbation vector $\mathbf{w}$ so that

$$
\{\hat{\mathbf{x}}, \mathbf{E}, \mathbf{w}\}=\arg \min _{\mathbf{x}, \mathbf{E}, \mathbf{w}}\|\mathbf{D}\|_{F}^{2}
$$

subject to

$$
\left(\mathbf{A}_{0}+\mathbf{E}\right) \mathbf{x}=\mathbf{b}_{0}+\mathbf{w}
$$

where the subscript $\mathrm{F}$ denotes the Frobenius norm.

The TLS optimization involves to find the optimum $\hat{\boldsymbol{\alpha}}$ for $\boldsymbol{\alpha}$ that minimizes the cost function $f(\hat{\boldsymbol{\alpha}})$,

$$
f(\hat{\boldsymbol{\alpha}})=\left\|\mathbf{B}_{0} \hat{\boldsymbol{\alpha}}\right\|_{F}^{2}=\hat{\boldsymbol{\alpha}}^{T} \mathbf{B}_{0}^{T} \mathbf{B}_{0} \hat{\boldsymbol{\alpha}}
$$

In practical applications, the value of matrix $\mathbf{B}_{0}$ is unknown. We now make a singular value decomposition (SVD) of the matrix $\mathbf{B}=[-\mathbf{b} \mathbf{A}]$, that is,

$$
\mathbf{B}=\mathbf{U}_{\mathrm{B}} \boldsymbol{\Sigma}_{\mathrm{B}} \mathbf{V}_{B}^{H}
$$

where $\boldsymbol{\Sigma}_{\mathrm{B}}$ is composed of the singulars value of $\mathbf{B}$. To solve (11), matrix $\mathbf{B}_{0}$ can be replaced by a rank three optimum approximation of $\mathbf{B}_{0}$, that is,

$$
\tilde{\mathbf{B}}=\mathbf{U}_{\mathrm{B}} \boldsymbol{\Sigma}_{3} \mathbf{V}_{B}^{H}
$$

where $\boldsymbol{\Sigma}_{3}$ is composed of the maximum three singulars value of $\mathbf{B}$. Now we have the cost function

$$
f(\hat{\boldsymbol{\alpha}})=\hat{\boldsymbol{\alpha}}^{T} \tilde{\mathbf{B}}^{T} \tilde{\mathbf{B}} \hat{\boldsymbol{\alpha}}
$$

The parameters in vector $\hat{\boldsymbol{\alpha}}$ are subject to a quadratic constraint 


$$
1+\left(x_{1}-\hat{x}_{\mathrm{s}}\right)^{2}+\left(y_{1}-\hat{y}_{\mathrm{s}}\right)^{2}-\hat{r}_{1}^{2}-1=0
$$

or, equivalently, as

$$
\hat{\boldsymbol{\alpha}}^{\mathrm{T}} \boldsymbol{\Sigma} \hat{\boldsymbol{\alpha}}-1=0
$$

where $\boldsymbol{\Sigma}=\operatorname{diag}(1,1,1,-1)$ is a diagonal and orthonormal matrix. The technique of Lagrange multipliers is used and the modified cost function is of the form

$$
\begin{aligned}
f(\hat{\boldsymbol{\alpha}}) & =\hat{\boldsymbol{\alpha}}^{T} \tilde{\mathbf{B}}^{T} \tilde{\mathbf{B}} \hat{\boldsymbol{\alpha}}+\lambda\left\{\hat{\boldsymbol{\alpha}}^{T} \boldsymbol{\Sigma} \hat{\boldsymbol{\alpha}}-1\right\} \\
& =\hat{\boldsymbol{\alpha}}^{T}\left(\tilde{\mathbf{B}}^{T} \tilde{\mathbf{B}}+\lambda \boldsymbol{\Sigma}\right) \hat{\boldsymbol{\alpha}}-\lambda
\end{aligned}
$$

where $\lambda$ is the Lagrange multiplier.

The required optimum parameter vector $\hat{\boldsymbol{\alpha}}$ is found by solving the following linear system of equations [12]

$$
\left(\tilde{\mathbf{B}}^{T} \tilde{\mathbf{B}}+\lambda \boldsymbol{\Sigma}\right) \hat{\boldsymbol{\alpha}}=k \mathbf{e}_{1}
$$

where, $\mathbf{e}_{1}=\left[\begin{array}{llll}1 & 0 & 0 & 0\end{array}\right]^{T}$, and normalizing constant $k$ is selected so that the first element of solution vector $\hat{\boldsymbol{\alpha}}$ is equal to one. Solving (18) is very easy, and it is independent of normalization constant $k$, which is unknown. Calculating the inverse of matrix $\left(\tilde{\mathbf{B}}^{T} \tilde{\mathbf{B}}+\lambda \boldsymbol{\Sigma}\right)$ and then normalizing the first element of $\hat{\boldsymbol{\alpha}}$, we can obtain the solution vector. The QC-TLS solution vector to (9) is

$$
\hat{\boldsymbol{\alpha}}=\frac{\left(\tilde{\mathbf{B}}^{T} \tilde{\mathbf{B}}+\lambda \boldsymbol{\Sigma}\right)^{-1} k \mathbf{e}_{1}}{\mathbf{e}_{1}^{T}\left(\tilde{\mathbf{B}}^{T} \tilde{\mathbf{B}}+\lambda \boldsymbol{\Sigma}\right)^{-1} k \mathbf{e}_{1}}=\frac{\left(\tilde{\mathbf{B}}^{T} \tilde{\mathbf{B}}+\lambda \boldsymbol{\Sigma}\right)^{-1} \mathbf{e}_{1}}{\mathbf{e}_{1}^{T}\left(\tilde{\mathbf{B}}^{T} \tilde{\mathbf{B}}+\lambda \boldsymbol{\Sigma}\right)^{-1} \mathbf{e}_{1}}
$$

The estimated $\mathrm{x}$ and $\mathrm{y}$ coordinates of the source are

where

$$
\begin{aligned}
& \hat{x}_{s}=\hat{\boldsymbol{\alpha}}^{T} \mathbf{e}_{2}+x_{1} \\
& \hat{y}_{s}=\hat{\boldsymbol{\alpha}}^{T} \mathbf{e}_{3}+y_{1}
\end{aligned}
$$

$$
\begin{aligned}
& \mathbf{e}_{2}=\left[\begin{array}{llll}
0 & 1 & 0 & 0
\end{array}\right]^{T} \\
& \mathbf{e}_{3}=\left[\begin{array}{llll}
0 & 0 & 1 & 0
\end{array}\right]^{T}
\end{aligned}
$$

Lagrange Multiplier $\lambda$ in the expression of solution vector $\hat{\boldsymbol{\alpha}}$ is unknown. In order to find $\lambda$, we can impose the quadratic constraint directly by substituting (19) into (16), so that

$$
\left[\frac{\left(\tilde{\mathbf{B}}^{T} \tilde{\mathbf{B}}+\lambda \boldsymbol{\Sigma}\right)^{-1} \mathbf{e}_{1}}{\mathbf{e}_{1}^{T}\left(\tilde{\mathbf{B}}^{T} \tilde{\mathbf{B}}+\lambda \boldsymbol{\Sigma}\right)^{-1} \mathbf{e}_{1}}\right]^{T} \boldsymbol{\Sigma}\left[\frac{\left(\tilde{\mathbf{B}}^{T} \tilde{\mathbf{B}}+\lambda \boldsymbol{\Sigma}\right)^{-1} \mathbf{e}_{1}}{\mathbf{e}_{1}^{T}\left(\tilde{\mathbf{B}}^{T} \tilde{\mathbf{B}}+\lambda \boldsymbol{\Sigma}\right)^{-1} \mathbf{e}_{1}}\right]-1=0
$$

By using an eigenvalue factorization, the matrix $\tilde{\mathbf{B}}^{T} \tilde{\mathbf{B}} \boldsymbol{\Sigma}$ can be diagonalized as

$$
\tilde{\mathbf{B}}^{T} \tilde{\mathbf{B}} \boldsymbol{\Sigma}=\mathbf{U} \boldsymbol{\Lambda} \mathbf{U}^{-1}
$$

where $\boldsymbol{\Lambda}=\operatorname{diag}\left(\gamma_{1}, \gamma_{2}, \gamma_{3}, \gamma_{4}\right), \gamma_{i}, i=1, \ldots, 4$ are the eigenvalues of the matrix $\tilde{\mathbf{B}}^{T} \tilde{\mathbf{B}} \boldsymbol{\Sigma}$, and $\mathbf{U}$ is the corresponding eigenmatrix of $\tilde{\mathbf{B}}^{T} \tilde{\mathbf{B}} \boldsymbol{\Sigma}$. Substituting (22) into (21) produces

$$
\frac{\mathbf{e}_{1}^{T} \boldsymbol{\Sigma} \mathbf{U}(\boldsymbol{\Lambda}+\lambda \mathbf{I})^{-2} \mathbf{U}^{-1} \mathbf{e}_{1}}{\left[\mathbf{e}_{1}^{T} \boldsymbol{\Sigma} \mathbf{U}(\boldsymbol{\Lambda}+\lambda \mathbf{I})^{-1} \mathbf{U}^{-1} \mathbf{e}_{1}\right]^{2}}-1=0
$$

For notational convenience, we define

$$
\begin{aligned}
\mathbf{\Sigma} & =\left[\begin{array}{llll}
\mathbf{p}_{1}^{T} & \mathbf{p}_{2}^{T} & \mathbf{p}_{3}^{T} & \mathbf{p}_{4}^{T}
\end{array}\right]^{T} \\
\mathbf{p}_{i} & =\left[\begin{array}{llll}
p_{i 1} & p_{i 2} & p_{i 3} & p_{i 4}
\end{array}\right] \\
\mathbf{U}^{-1} & =\left[\begin{array}{llll}
\mathbf{q}_{1} & \mathbf{q}_{2} & \mathbf{q}_{3} & \mathbf{q}_{4}
\end{array}\right] \\
\mathbf{q}_{i} & =\left[\begin{array}{llll}
q_{1 i} & q_{2 i} & q_{3 i} & q_{4 i}
\end{array}\right]^{T}
\end{aligned}
$$

Substituting (24) into (23), then the quadratic constraint in the form of $\lambda$ becomes

$$
\begin{aligned}
f(\lambda)= & \sum_{i=1}^{4} \frac{p_{1 i} q_{i 1}}{\left(\gamma_{i}+\lambda\right)^{2}} \cdot \prod_{i=1}^{4}\left(\gamma_{i}+\lambda\right)^{2} \\
& -\left(\sum_{i=1}^{4} \frac{p_{1 i} q_{i 1}}{\gamma_{i}+\lambda} \cdot \prod_{i=1}^{4}\left(\gamma_{i}+\lambda\right)\right)^{2}=0
\end{aligned}
$$

Lagrange Multiplier $\lambda$ can be solved from (25) by using Newton's method with zero as the initial guess.

To sum up, a brief description of the proposed TDOA based location algorithm is as follows:

1) Calculate the optimum approximation of augmented matrix $\mathbf{B}_{0}$ using (12) and (13).

2) Solve $\lambda$ by finding the root of (25) using Newton's method.

3) Obtain the QC-TLS solution to (9) using (19) and determine $\mathrm{x}$ and $\mathrm{y}$ coordinates of the source using (20).

\section{Simulations}

We have proposed a QC-TLS algorithm for TDOA based location problem. In this section, we evaluate its performance at practical measurement error levels using Monte-Carlo simulations.

Source and sensors were located in random positions in a square of area $100 \times 100 \mathrm{~m}^{2}$ as shown in Fig. 2. We assumed that the TDOA measurement errors were white random processes with zero mean and variance $\sigma_{\mathrm{TDOA}}^{2}$, and the TDOA variance of all sensor inputs were identical. For simplicity, the TDOA variance was translated into corresponding distance variance $\sigma^{2}=\left(c \sigma_{\mathrm{TDOA}}\right)^{2}$. Simulation results provided were averages of 10000 independent runs. We compare the proposed QC-TLS approach with OLS approach and CRLB. 
Tables 1 and 2 compare the mean absolute location errors (MALEs) of OLS and QC-TLS approach for various TDOA error variances, and MALE was defined as $E\left[\sqrt{\left(x_{s}-\hat{x}_{s}\right)^{2}+\left(y_{s}-\hat{y}_{s}\right)^{2}}\right]$. The number of sensors was set to 7 and 10 for Table 1 and Table 2, respectively. In Tables 1 and 2, CRLB, which is a fundamental lower bound on the variance of any unbiased estimator, is calculated using the functions in [4]. The distance unit is meters. We observe that increasing the number of sensors can increase the location accuracy, because increasing the number of sensors can provide more redundant information which could be helpful for solving the overdetermined linear equations.

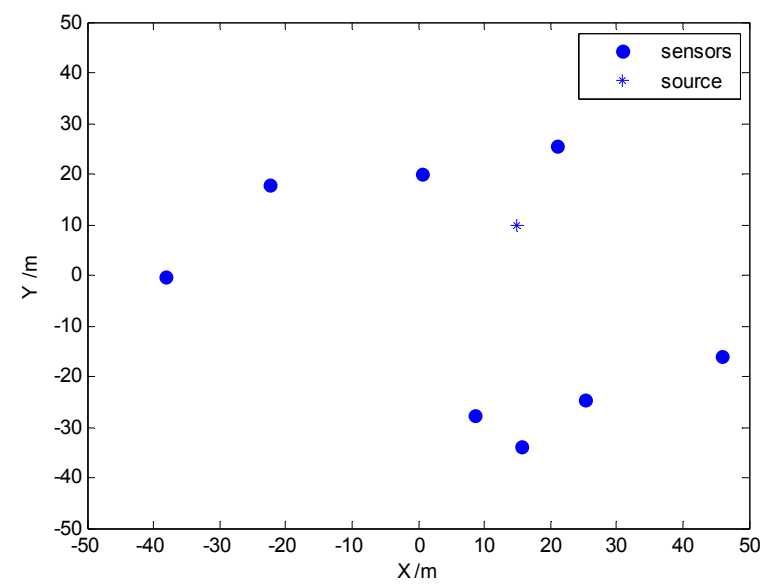

Figure 2. Location in 2-D plane

Table 1. comparison of MALEs for QC-TLS and OLS method for various variances: seven sensors

\begin{tabular}{ccccc}
\hline$\sigma^{2}$ & 0.1 & 0.01 & 0.001 & 0.0001 \\
\hline OLS & 0.9359 & 0.2962 & 0.0939 & 0.0293 \\
QC-TLS & 0.7735 & 0.2236 & 0.0709 & 0.0221 \\
CRLB & 0.6706 & 0.2134 & 0.0677 & 0.0214 \\
\hline
\end{tabular}

Table 2. comparison of MALEs for QC-TLS and OLS method for various variances: ten sensors

\begin{tabular}{ccccc}
\hline$\sigma^{2}$ & 0.1 & 0.01 & 0.001 & 0.0001 \\
\hline OLS & 0.5801 & 0.1851 & 0.0571 & 0.0182 \\
QC-TLS & 0.5410 & 0.1501 & 0.0472 & 0.0150 \\
CRLB & 0.4280 & 0.1361 & 0.0428 & 0.0136 \\
\hline
\end{tabular}

The performance of QC-TLS approach is much better than OLS approach especially when the TDOA variance is large, and it is closer to CRLB. They verify that the proposed approach could inhibit the influence of TDOA measurement errors on the estimation results better than OLS. When TDOA variance is large, the noise in matrix A, which would be alleviated by QC-TLS approach, could significantly reduce the performance of OLS approach.
Mean absolute location error is one of the metrics to evaluate the performance of QC-TLS algorithm, and computational complexity is another important metric to evaluate the performance of algorithms. The price of the better performance for QC-TLS is that its computational complexity is larger than OLS. In the same simulation condition, the time of 10000 independent runs for QCTLS and OLS are approximately $6153.6 \mathrm{~ms}$ and 1446.3 $\mathrm{ms}$, respectively. It is obviously that QC-TLS algorithm requires matrix singular value decomposition once, eigenvalue decomposition once, Newton iteration once, inverse operation once and matrix multiply operation several times, whereas OLS algorithm only requires matrix pseudoinverse operation once and matrix multiply operation several times. It's noteworthy that QC-TLS algorithm performs better at a cost of larger computational complexity.

\section{Conclusions}

A novel TDOA based quadratic constraint total leastsquares location algorithm is proposed in this paper. The proposed algorithm utilizes TLS to inhibit the influence of TDOA measurement errors. And the technique of Lagrange multipliers is utilized to exploit the quadratic constraint relation between the intermediate variables to constrain the solution to the location equations and improve the location accuracy. Simulation results indicate that the proposed QC-TLS algorithm gives better results than the OLS solution.

\section{References}

[1] N. Patwari, J. N. Ash, S. Kyperountas, A. O. Hero III, R. L. Moses, and N. S. Correal, "Locating the Nodes: Cooperative Localization in Wireless Sensor Networks," IEEE Signal Processing Magazine, vol. 22, no. 4, pp. 5468, July 2005.

[2] X. Jun, L. R. Ren, and J. D. Tan, "Research of TDOA based self-localization approach in wireless sensor network," in proceedings of IEEE International Conference on Intelligent Robots and Systems, Beijing, pp. 2035-2040, October 2006.

[3] J. Smith and J. Abel, "Closed-form least-squares source location estimation from range-difference measurements," IEEE Transactions on Acoustics, Speech, and Signal Processing, vol. 35, pp. 1661-1669, December 1987.

[4] Y. T. Chan and K. C. Ho, "A simple and efficient estimation for hyperbolic location," IEEE Transactions on Signal processing, vol. 42, no. 8, pp. 1905-1915, August 1994.

[5] R. Schmidt, "Least squares range difference location," IEEE Transactions on Aerospace and Electronic Systems, vol. 32, no. 1, pp. 234-242, January 1996.

[6] Y. Huang, J. Benesty, G. W. Elko, and R. M. Mersereau, "Real-time passive source localization: a practical linearcorrection least-squares approach," IEEE Transactions 
FOR HYPERBOLIC LOCATION

On Speech And Audio Processing, vol. 9, no. 8, pp. 943956, November 2001.

[7] T. J. Abatzoglou, J. M. Mendel, and G. A. Harada, "The constrained total least squares technique and its applications to harmonic superresolution," IEEE Transactions on Signal Processing, vol. 39, no. 5, pp. 1070-1087, May 1991.

[8] S. V. Huffel and J. Vandewalle, "The Total Least Squares Problem: Computational Aspects and Analysis," Philadelphia: SIAM, 1991.

[9] I. Markovsky and S. V. Huffel, "Overview of total leastsquares methods," Signal Processing, vol. 87 no. 10, pp.
2283-2302, October 2007.

[10] X. Li, "Super-Resolution TOA Estimation with Diversity Techniques for Indoor Geolocation Applications," Ph.D. Dissertation, Worcester Polytechnic Institute, Worcester, MA, 2003.

[11] S. D. Hodges and P. G. Moore, "Data Uncertainties and Least Squares Regression,” Applied Statistics, vol. 21, pp. 185-195, 1972.

[12] J. A. Cadzow, "Spectral estimation: An overdetermined rational model equation approach," Proceedings of the IEEE, vol. 70, no. 9, pp.907-939, September 1982. 